\title{
Safe and cost-effective rapid-prototyping of multilayer PMMA microfluidic devices
}

\author{
Antonio Liga ${ }^{1,2} \cdot$ Jonathan A. S. Morton ${ }^{3}$ Maïwenn Kersaudy-Kerhoas ${ }^{1,2}$ (])
}

Received: 10 August 2016 / Accepted: 3 November 2016 / Published online: 25 November 2016

(C) The Author(s) 2016. This article is published with open access at Springerlink.com

\begin{abstract}
The rapid translation of research from bench to bedside, as well as the generation of commercial impact, has never been more important for both academic and industrial researchers. It is therefore unsurprising that more and more microfluidic groups are investigating research using a wide range of thermoplastics which can be readily translated to large-scale manufacturing, if the technology is taken to commercialisation. While structuring, via additive or subtractive manufacturing, is becoming relatively easy through the use of commercial-grade devices, reliable and fast assembly remains a challenge. In this article, we propose an innocuous and cost-effective, under 2-min technique which enables the bonding of multiple poly(methyl methacrylate) layers. This bonding technique relies on the application of small amounts $\left(10 \mu \mathrm{l} / \mathrm{cm}^{2}\right)$ of ethanol, low temperatures $\left(70{ }^{\circ} \mathrm{C}\right)$ and relatively low pressures $(\sim 1.6 \mathrm{MPa})$. Our characterisation analysis shows that using a bonding time of $2 \mathrm{~min}$ is enough to produce a strong bond able to withstand pressures always above 6.2 $\mathrm{MPa}$ (with mean of $8 \mathrm{MPa}$, highest reported in the
\end{abstract}

Electronic supplementary material The online version of this article (doi:10.1007/s10404-016-1823-1) contains supplementary material, which is available to authorized users.

Maïwenn Kersaudy-Kerhoas

m.kersaudy-kerhoas@hw.ac.uk

1 Institute of Biological Chemistry, Biophysics and Bioengineering, School of Engineering and Physical Science, Heriot-Watt University, Edinburgh, UK

2 Division of Infection and Pathway Medicine, University of Edinburgh, Edinburgh, UK

3 Institute of Photonics and Quantum Sciences, School of Engineering and Physical Science, Heriot-Watt University, Edinburgh, UK literature), with minimal channel deformation $(<5 \%)$. This technique, which we demonstrate on assembly comprising up to 19 layers, presents an exciting improvement in the field of rapid prototyping of microfluidic devices, enabling extremely fast design cycles in microfluidic research with a material compatible with mass manufacturing, thus allowing a smoother transition from the laboratory to the market. Beyond the research community, this robust prototyping technique has the potential to impact on the deliverability of other scientific endeavours including educational projects and will directly fuel the fluidic maker movement.

\section{Introduction}

Due to its low cost, optical transparency and the availability of a range of processing methods, poly(methyl methacrylate) (PMMA) has been adopted for many microfluidic applications (Das et al. 2015; Pagaduan et al. 2015). Compared to silicon-based materials used for microfluidic applications, PMMA is less fragile and does not require expensive manufacturing techniques. PMMA is therefore preferred, together with plastics like polystyrene, polycarbonate and cyclic olefin copolymer, for the manufacturing of disposable devices required for commercially orientated applications (Romoli et al. 2011). PMMA structures can be created by a wide range of techniques including hot embossing, injection moulding, laser ablation, reactive ion etching or deep UV lithography and have mechanical properties superior to those of poly(dimethylsiloxane) (PDMS, another material of choice for microfluidic applications), guaranteeing a better fidelity to the initial shape under mechanical stress conditions (Madadi et al. 2013). However, sealing of PMMA devices is not as straightforward as that of more conventional materials used traditionally in 
microfluidic research, such as PDMS, and mostly relies on methodologies that can lead to severe changes in the morphology of the final product (Tran et al. 2013). The need of a reliable and high-throughput bonding method becomes critical as applications are rapidly translated into commercialised products, in particular for clinical products (Tsao and DeVoe 2009; Becker 2010). Additionally, over the last 5 years small milling machines and table-top laser systems have become available and form, besides 3D printing, the bases for the maker movement. These structuring techniques can also be used for generating microfluidic structures well suited for the education of students and for prototyping at many places around the world backed by networks such as the Fab Lab network (Nascimento and Pólvora 2016). However, up to now, no satisfying bonding technique has been developed. Double-sided adhesive tape is often used for convenience and price, but has a number of drawbacks including poor optical qualities and chemical incompatibilities. In this work, we show a bonding method that allows for the fast, cost-effective and safe bonding of multilayer stacks of PMMA, thus overtaking part of the barrier that prevents the translation of microfluidics from the laboratory to the real world. The most common PMMA bonding technique is thermal bonding, in which PMMA elements are heated above the glass transition temperature $\left[T_{\mathrm{g}}\right.$ ranging from $85{ }^{\circ} \mathrm{C}$ to $165{ }^{\circ} \mathrm{C}$ depending on the commercial composition (Duan et al. 2010) but usually around $110{ }^{\circ} \mathrm{C}$ ( $\mathrm{Li}$ et al. 2015)] and pressed together. During thermal bonding, the diffusion of polymer chains between the two interfaces leads to the formation of entanglements among the macromolecules (Baldan 2012) and permanent bonding. The influence of PMMA molecular weight on thermal bonding was studied, showing that longer macromolecules can form stronger bonds (Nayak et al. 2009). Thermal bonding protocols often include temperatures up to $140{ }^{\circ} \mathrm{C}$ at the pressing plates, to ensure that the whole PMMA artefact is above the PMMA glass transition temperature $\left(T_{\mathrm{g}}\right)$. To avoid thermally induced stress that would result in layers bending, the material needs to reach the final temperature slowly and similarly requires a slow cooling. Therefore, the entire process can last for several hours (Zhu et al. 2006; Mohammed et al. 2013). Widely used due to its simplicity, this technique does, however, result in the distortion of the PMMA elements. More elaborate solutions to alleviate this distortion issue have been devised, but are not free from drawbacks. Intermediate adhesive layers have been proposed, but can interact with the fluid passing through the microfluidic channels. Surface modification and solvent-assisted bonding (Brown et al. 2006) which can change surface shape but usually involve highly toxic chemicals (chloromethane, dichloromethane, chloroform) (Jiang et al. 2015) are other proposed methods. Furthermore, both methods can lead to clogging of the channels
(Lin et al. 2007). Variants of the aforementioned solutions include the use of UV light to activate surfaces (Tran et al. 2013), microwave heating combined with a conductive layer [patterned gold (Toossi et al. 2015), conductive polymer (Holmes et al. 2011) or metal clips acting on a solvent layer (Mona et al. 2010)], or plasma treatment (Cheng et al. 2015), which can be optimised to reach a bonding strength of $0.57 \mathrm{MPa}$ in $20 \mathrm{~min}$ (Zhifu et al. 2015). Generally these procedures are time-consuming and require specialist equipment (Lin et al. 2007). It was shown that containment grooves between the layers to bond can improve the efficiency of solvent-assisted bonding methods (Wan et al. 2015). Interfacial heating caused by low amplitude ultrasonic was used to bind preheated PMMA layers with imprinted nanochannels (Zhang et al. 2010). With the use of plasticisers, bonding can be performed at lower temperatures by lowering the glass transition of the material. Low deformation $(<10 \%)$ low temperature $\left(90^{\circ} \mathrm{C}\right)$ PMMA bonding was achieved using dibutyl phthalate (DBP) as a plasticiser (Duan et al. 2010). Nonetheless, DBP is a suspected teratogen banned in many countries, and its presence within the bulk of the final artefact could make the bonding step unsafe and prevent its use in medical applications. A fast PMMA prototyping methodology, developed by Sun et al. (2007), consists of a solvent-based imprinting and bonding strategy. This method allows for the realisation of PMMA chips in 15 min; nonetheless, it requires the use of photolithography techniques for the realisation of a template for the imprinting in a photoresist material, commonly SU8. Custom devices were built so as to perform thermal bonding in line with injection moulding, so as to save processing time and thermal energy. This method is fast and reliable, but requires a custom device and only allows the bonding of two layers, thus significantly limiting chip design (Chu et al. 2015). Using layers of a lower $T_{\mathrm{g}}$ polymer, such as TMMF (photosensitive epoxy laminate) for the thermal welding of PMMA, reduces deformation on the PMMA bulk, although the procedure is time-consuming and requires photolithography equipment for the shaping of TMMF (Kalkandjiev et al. 2010).

We propose an optimised solvent-assisted thermal bonding method, allowing for cost-effective, innocuous and ultrafast bonding. The solvent used is absolute ethanol, which is nontoxic, does not require to be handled under a fume hood in the small amounts required for the process and is extremely cheap $(\sim 5 / \mathrm{L})$. This method is therefore ideal for fast prototyping and manufacturing, and its use might encourage the use of PMMA, a material more easily adapted to industrial translation than PDMS, in microfluidic research settings. This technique might also be of interest to the so-called Maker movement and the burgeoning microfluidic "spin-off" of this movement (Frot et al. 2016; Silva et al. 2015). The concept of ethanol-assisted thermal 


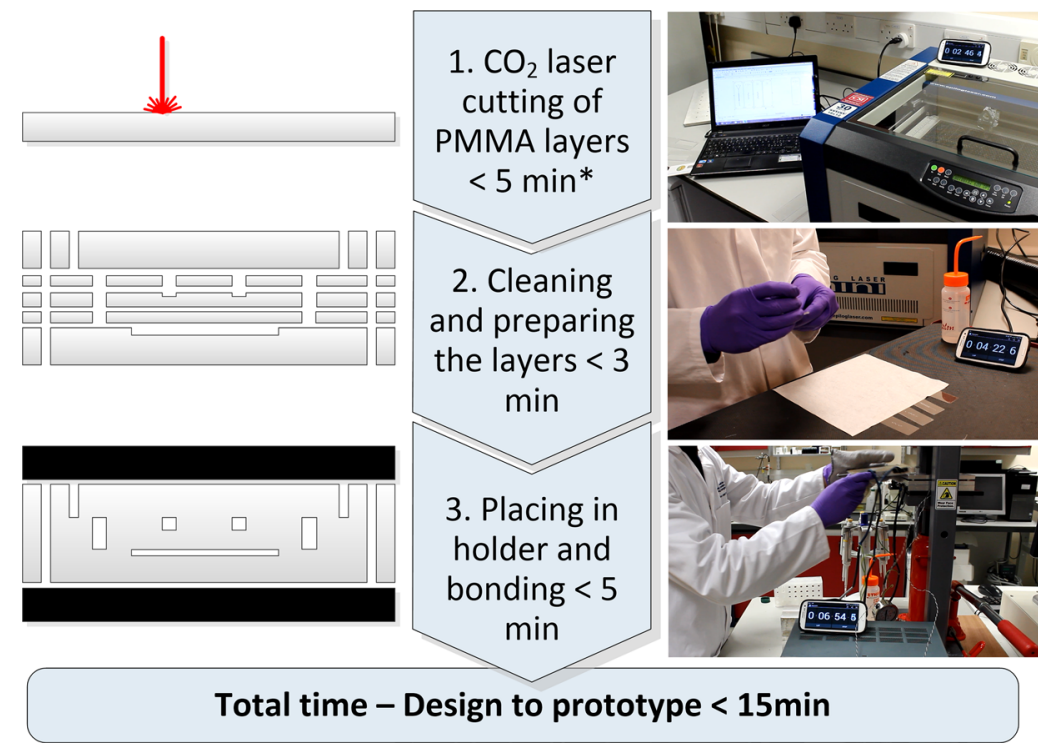

\begin{tabular}{|c|c|c|}
\hline 鼔 & $\begin{array}{l}\text { Cutting Equipment } \\
\text { Bonding Equipment } \\
\text { TOTAL }\end{array}$ & $\begin{array}{l}<\mathrm{k} \$ 14 \\
<\mathrm{k} \$ 1.2 \\
<\mathrm{k} \$ 16\end{array}$ \\
\hline 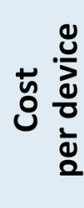 & $\begin{array}{l}\text { Material } \\
\text { Electricity } \\
\text { Ethanol } \\
\text { Yearly maintenance** } \\
\text { TOTAL }\end{array}$ & $\begin{array}{l}\$ 1.78 \\
\$ 0.01 \\
\$ 0.001 \\
\$ 0.1 \\
\$ 1.89\end{array}$ \\
\hline 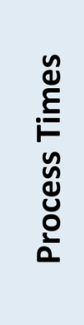 & $\begin{array}{l}\text { Device fabrication } \\
\text { Machine Setup } \\
\text { Material Setup } \\
\text { Cutting* } \\
\text { Device assembly/bonding } \\
\text { Machine/Material Setup } \\
\text { Preparation and bonding } \\
\text { TOTAL }\end{array}$ & $\begin{array}{l}1 \mathrm{~min} \\
1 \mathrm{~min} \\
4 \mathrm{~min}^{*} \\
3 \mathrm{~min} \\
5 \mathrm{~min} \\
<15 \mathrm{~min}\end{array}$ \\
\hline
\end{tabular}

Fig. 1 Ultrafast prototyping scheme, cost factors and process times. The *step timing is directly depending on the complexity of the device design. We demonstrate a laser cutting time of $4^{\prime} 09^{\prime \prime}$ for a five-layer microfluidic mixer unit with the size of typical microscope slide (Suppl Info video). **Yearly maintenance cost per device is estimated at $\$ 500$ per year divided by the production of 5000 units a year. ***This is based on a $\$ 800$ manual hydraulic press and a $\$ 400$ custom-made thermal controller (Suppl. Inf Table 1) bonding has been described before (Klank et al. 2002) and is driven by the need for maintaining the initial shape of every layer involved in the bonding process. Indeed, by acting at a temperature at which only the ethanol-wet surfaces are modified, it is possible to minimise the effect on the bulk of the material. Ethanol acts both as a solvent (we noticed deposits of PMMA in the channels when preliminarily testing with large amounts of ethanol) and as a plasticiser, as it was shown (Wang et al. 1994) that it lowers the $T_{\mathrm{g}}$ of PMMA to $11^{\circ} \mathrm{C}$, thus allowing the movement of polymer chains at the interfaces. Yang described the characterisation of postembossing ethanol bonding technique lasting a total of more than $6 \mathrm{~h}$ (Yang 2014). In another example, Hsu and Chen explored bonding with ethanol, methanol and isopropanol using a Taguchi approach for experiments planning (Hsu and Chen 2007). However, as the Taguchi method aims to reduce the amount of total experiments required by adding together the main effects of independent variables, it was not applicable to this study and led to misleading results in optimisation terms. Indeed, solvent type, temperature and bonding time cannot be considered as independent variables and their effects are not separable, as the physics ruling the bonding process (solubilisation of the polymer chains, diffusion of solvent within the polymer matrix) follows kinetics in which the solvent molecules/ polymer interactions are strictly connected to temperature (Santos et al. 2016; Bird et al. 2002; Green and Perry 2008). Also, bonding strength is not a monotonic function of bonding pressure (as we demonstrate), another assumption that needs to be respected in order to use the Taguchi method. To the best of our knowledge, we demonstrate for the first time that a bonding time of only $2 \mathrm{~min}$ is needed to achieve crack-free structures with a reliable bonding strength, furthermore using a cost-effective and safe solvent and a readily available experimental set-up costing under $\$ 1200$. As our example of prototyping method, we chose laser cutting (Fig. 1) as a first step, in order to produce the different layers out of PMMA sheets with different thicknesses; nonetheless, other layer shaping techniques can be coupled with the bonding method described herein. Bonding stability was tested by means of quickened ageing in an oven at $90{ }^{\circ} \mathrm{C}$ and in water incubation at $37.5^{\circ} \mathrm{C}$. The application of rapid prototyping methods from structuring to sealing is well justified and may alleviate issues encountered in the translation to production (Hardt et al. 2005). Together with its rapidity, another great advantage of this method lies in the possibility to assemble several layers, thus allowing the formation of complex 3D microfluidic structures.

\section{Materials and methods}

\subsection{PMMA bonding}

PMMA sheets of $0.2,0.8$ and $2.0 \mathrm{~mm}$ thicknesses acquired from various suppliers were used in this study. Bonding optimisation studies, as well as exemplary chips (Sect. 3.6), were realised with Clare ${ }^{\circledR}$ (Nitto Jushi Kogyo Co. Ltd, JP) cast PMMA sheets supplied by Weatherall Ltd, UK. For 
material comparison, Oroglas ${ }^{\circledR}$ (Oroglas Ltd, FR) and Per$\operatorname{spex}^{\circledR}$ (Lucite International Ltd) cast sheets were obtained, respectively, from Easter Road Plastics Ltd, UK, and Engineering and Design Plastics Ltd, UK. Extruded Perspex ${ }^{\circledR}$, supplied by Engineering and Design Plastics Ltd, UK, was also included in the material comparison study (for material details, c.f. Table S1 Suppl. Inf). Absolute Ethanol (\%w $>99.95 \% \mathrm{w})$ was used as a solvent (Sigma-Aldrich, UK). A custom-made heating plate and control unit were developed to heat up and maintain the layers at the correct temperature during bonding (c.f. Suppl. Inf. Table S.2). As a source of pressure, we used a Bonny Doon Classic 20-Ton Manual Press (Rio Grande, USA) or a manual 30 tons hydraulic pellet press (Research \& Industrial Instrument Company, UK). Results obtained from both presses are compared in the results and discussion section.

After laser cutting the desired shapes using a $\mathrm{CO}_{2}$ laser cutter (Epilog Mini 18, Epilog, USA), PMMA layers were cleaned with clean-room tissue and ethanol to remove dust. In this step, the minute amount of ethanol applied to the layers via the tissue evaporates in a few seconds after application and does not affect the bonding subsequently. Ethanol was spread between the layers with a pipette just before bonding ( $20 \mu \mathrm{l}$ for shear stress test specimens, $80 \mu \mathrm{l}$ for deformation specimens, burst test specimens and our application examples). This allows for the partial melting of a superficial layer and the formation of a strong bond between the PMMA elements even if operating below PMMA $T_{\mathrm{g}}$.

In Fig. 1, the workflow of the process is shown together with economic considerations on equipment and maintenance. Layers were aligned in holders specific for each of the sample types. When several layers needed to be aligned with precision, such as in the realisation of complex microfluidic platforms, custom-made plates with pins for alignment of the layers are preferred; therefore, individual layers were featured with corresponding alignment holes. For more simple geometries, custom frames were laser cut in PMMA. As the thermal profile within the PMMA layer stack to bind is dependent on its thickness, all the specimens for shear stress, burst and deformation analysis were realised in stacks $4-4.2 \mathrm{~mm}$ tall. The microfluidic device examples shown in Sect. 3.6 have thickness ranging from 2.4 to $5.2 \mathrm{~mm}$ and showed high performances without the need of further tuning of the bonding parameters. For PMMA stacks taller than $6 \mathrm{~mm}$, a longer bonding time than the one suggested in this paper will be required.

\subsection{Shear stress analysis}

Shear stress testing was performed by means of a tensile test equipment (Instron 3367, Instron, UK) with an elongation speed of $0.05 \mathrm{~mm} / \mathrm{s}$ and recording the highest load before sample break. The two sides of T-bones specimens with no microfluidic features were laser cut from 2.0-mmthick sheets, and an additional layer was added with double-sided tape on the gripping side, to ensure alignment during bonding and shear stress test (c.f. Suppl Info Figure S.1). They were fixed in a custom holding frame, placed on the bottom heater plate, and $20 \mu$ of ethanol was pipetted within the overlapped area $\left(2 \mathrm{~cm}^{2}\right)$. The top heater plate was placed above the specimens before applying pressure using the hydraulic press. The influence of temperature was studied by keeping bonding pressure and time constant at 5 tons at the ram (2800 psi inside the cylinder/1.57 MPa on the specimen surface) for $120 \mathrm{~s}$. Tests were run for a range of temperatures between 50 and $110^{\circ} \mathrm{C}$. The effect of pressure was studied at a bonding temperature and time of $70{ }^{\circ} \mathrm{C}$ and $120 \mathrm{~s}$, respectively; $2,3,5,8$ and 12 tons at the ram were used during specimen preparation, corresponding to $0.63,0.94,1.57,2.51$ and $3.76 \mathrm{MPa}$ applied on the surface of the specimens. Finally, in order to analyse the influence of time on bonding strength, tests were performed at $70{ }^{\circ} \mathrm{C}$ and 5 tons at the ram for $10,30,60,120,300$ and $480 \mathrm{~s}$. Between 6 and 12 specimens were used for each set of parameters.

Statistical relevance was calculated with ANOVA tests and, when necessary, with an unpaired, two-tailed student's $t$ test (VassarStats/GraphPad software).

\subsection{Burst test analysis}

One of the most important requirements for a microfluidic bonding method is its ability to efficiently seal channels, in such a way that they can stand pressure without suffering from leaks. Therefore, burst test analysis was performed within the same range of bonding pressure, time and temperature used for shear stress testing. Considering the larger area to bind, $80 \mu \mathrm{l}$ of ethanol was spread between each of the layers. Chips featuring a 0.5 -mm-wide channel leading to a dead-end 4-mm-diameter round chamber were realised sandwiching a $0.2-\mathrm{mm}$-thick layer, between two 2.0-mm-thick PMMA layers, one of which served also for the fluidic connection (realised through ferrules and connectors, supplied by Epigem Ltd, UK, tightened to the chip by a screw/nut system). A Nemesys mid-pressure (up to $20 \mathrm{MPa}$ ) syringe pump (Cetoni Gmbh, DE), with a medium pressure stainless steel 5-ml syringe was used to push deionised water within the specimens at $0.5 \mathrm{ml} / \mathrm{min}$. As there is no way out, the air enclosed within the channel and chambers is gradually forced in a smaller volume and its pressure increases. Pressure changes were monitored with a Nemesys pressure sensor for measurement up to $20 \mathrm{MPa}$ (Cetoni) through a Nemesys user interface PC Software v20151214. The highest pressure reached before chip failure was considered the burst limit of the specimens. As 
just above $10 \mathrm{MPa}$ of pressure, leaks were noticed at the syringe pump and tubing connection, testing was stopped every time $10 \mathrm{MPa}$ was exceeded; thus, the pressure limit reported for some specimens is safely underestimated with respect to the actual resistance of the chip. At least three specimens were used for each bonding condition.

\subsection{Deformation analysis}

Tests were performed to study how bonding parameters (pressure, temperature and time) affect the shape of the channel. Rectangular $(81 \times 40 \mathrm{~mm})$ specimens were fabricated by laser cutting sheets of PMMA and sandwiching a 0.2 -mm-thick layer between two 2.0-mm-thick layers. To allow a direct comparison, the area of these rectangles was chosen in order to have the same pressure distribution sustained by the shear stress and burst test specimens when the same force is applied at the ram. In the central layer, three $0.5 \times 10 \mathrm{~mm}$ channels were laser cut. Before hot pressing the three layers together, $80 \mu \mathrm{l}$ of ethanol was pipetted between each of the layers and spread along the whole surfaces by gently rubbing them together. Tests were performed in the same range of bonding temperature, pressure and time as shear stress analysis. To check the different impact that bonding time may have below and above glass transition temperature, tests at 70 and $110{ }^{\circ} \mathrm{C}$ were also performed for $480 \mathrm{~s}$. Finally, an extreme case study was realised by bonding a specimen at $110{ }^{\circ} \mathrm{C}$ and 12 tons at the ram (3.76 MPa) for $480 \mathrm{~s}$.

Samples were prepared for cross-sectional analysis by cutting through the imbedded channels using a diamond saw. Imaging of the channel cross sections was carried out with a Leica DM6000 microscope with bright-field illumination in transmission mode. An overall magnification of $50 \mathrm{X}$ was used to record the images. For some specimens, a deformed area was noticed around the channels, probably due to solvent swelling. Both a channel height and a deformed area height were recorded. In order to compensate for the variations in the thickness of the central layer ( $200 \mu \mathrm{m} \pm 10 \%$ according to manufacturer), the recorded heights were divided by the initial measured thickness for each chip and data are reported as dimensionless normalised deformation.

\subsection{Ageing testing}

In order to test the stability of the bonding under long-term storage, 18 shear stress specimens were manufactured, starting with elements cut from the same 2.0-mm-thick PMMA sheet, bonding at $70{ }^{\circ} \mathrm{C}$ and 5 tons at the ram for 2 min (optimal conditions). Six specimens underwent shear stress testing within the next $24 \mathrm{~h}$ from the bonding step, being stored at room conditions. For accelerated ageing, six were incubated within a closed bottle filled with deionised water at $37{ }^{\circ} \mathrm{C}$ for $216 \mathrm{~h}$, before testing. Similarly, the last six were kept at $90{ }^{\circ} \mathrm{C}$ for $216 \mathrm{~h}$ before testing. Incubation steps were performed in a MK 53 Dynamic climate chamber (Binder GmbH, DE).

\section{Results and discussion}

In this part, results and discussions are separated in sections aimed to explain how pressure, time and temperature affect the bonding strength.

\subsection{Influence of pressure}

The bonding strength was shown to follow a nonmonotone trend by both shear and burst analysis (Fig. 2a). The plots report the load at break $(\mathrm{N})$. The shear stress at break $(\mathrm{N} /$ $\mathrm{m}^{2}$ ) was not reported because in most cases concentrated shear stresses at the bent interfaces cause normal stress failure. The plots show a clear trend, and in particular, a peak in bonding strength is present for specimens bonded pressing with 5 tons at the ram. In order to explain the inefficient bonding at low pressure, it is easy to invoke an incomplete adhesion between the layers to bind and, often, bubbleshaped unbonded areas were noticed for low pressure bonding. Regarding the behaviour shown at higher pressures, two possible scenarios can be considered. During preliminary testing, it was noticed that excessively high-pressure results in wrinkles in the PMMA layers. This could result in incomplete matching of the surfaces. Another way of interpreting the phenomenon is considering that, when pushing the two surfaces together, a higher pressure will result in a thinner film of ethanol spread in between the PMMA layers, thus reducing the effect of the solvent. It is worth noticing that all the results obtained with the 20 tons press, although showing exactly the same trend as those of the 30 tons press, always showed higher bonding strength. The ram of this press terminates in a square plate that fits perfectly the custom-made heater plates. On the contrary, the ram of the 30 tons press was round and, due to the shape of the piece of equipment, it was hard to place the heating system exactly at the centre of the pressure point. We decided to show both results, as they show the same trend as well as give an indication that a good alignment can guarantee a more even pressure distribution, resulting in stronger bonding.

Deformation does not seem to strictly depend on pressure, although at higher pressures (8-12 tons at the ram) cracks were often noticed around channels (Fig. 3a). The internal heights of the channels were shown to reduce between 1 and 3\% (sagging) while the deformed layer was $7-8 \%$ higher than channels. An increase in channel height 


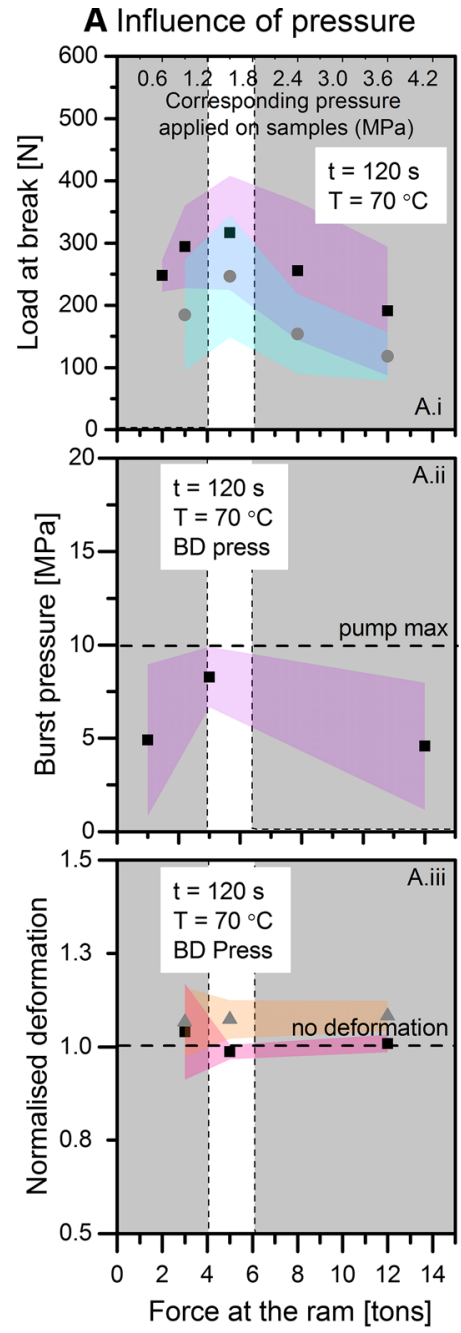

B Influence of time
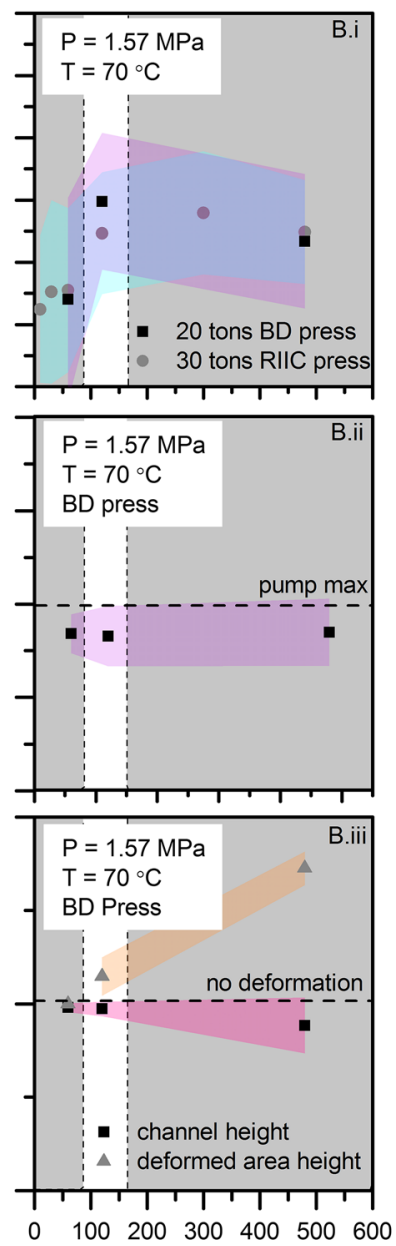

Time [s]
C Influence of temperature
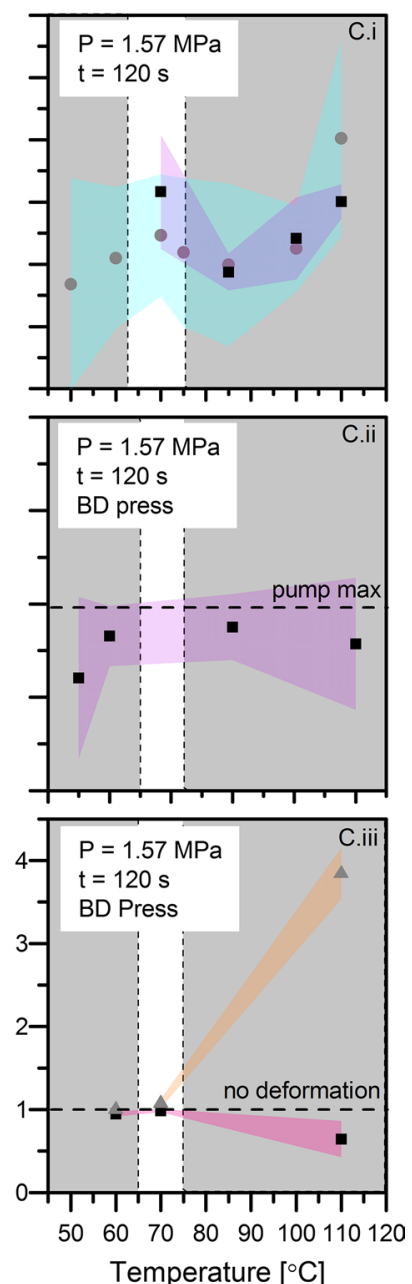

Fig. 2 Results for shear stress test (row 1$)(N=6-12)$, burst test (row 2) $(N=3)$ and deformation analysis (row 3$)(N=3-6)$ as a function of bonding pressure (column 1), time (column 2) and temperature (column 3). On all graphs, the vertical dash lines and white background zone highlight the optimal parameters. The SDs on all graphs are represented using coloured regions. The width of the region at a given data point of the graph gives the exact SD at this point. Between data points, the upper and lower SD is connected by a straight line to facilitate the reading of trends. Shear stress results (row 1) are shown for specimens bonded with both a 20 tons BD press (black squares) and a 30 tons RIIC press (grey circles). The 20 tons BD press was preferred for all subsequent experiments (including rows 2 and 3 and subsequent result sections). Hydraulic equivalents are given in the text. On the burst test (row 2) results, the dash horizontal line corresponds to the maximum value achieved by the Nemesys high-pressure equipment. For the deformation analysis (row 3), the plot reports the height of both the channels (which sag during harsher bonding conditions, black squares) and the deformed areas around channels (which on the contrary grow with harsher bonding conditions, grey triangles). For readability, the horizontal dash line indicates the absence of deformation. The collective reading of the three tests (shear stress, burst, deformation) against each of the parameters (pressure, time, temperature) gives a clear reading of the optimal parameters, highlighted by the white zones for each parameter was shown for $0.3 \mathrm{MPa}$ bonding, probably due to a slight solvent erosion effect $(\sim 10 \mu \mathrm{m})$ occurring at lower pressure, when the amount of ethanol constrained within the layers is reasonably higher.

\subsection{Influence of time}

Shear stress testing at $70{ }^{\circ} \mathrm{C}$ and 5 tons at the ram (1.57 $\mathrm{MPa}$ on specimens) showed that bonding strength is strongly dependent on bonding time for the first $120 \mathrm{~s}$.
After this, a plateau is reached and pressing the specimens for longer did not result in any advantage in terms of bonding strength. On the other hand, not all of the specimens processed for times shorter than $60 \mathrm{~s}$ were completely bonded (Fig. 2b.i), and sometimes bonding failure occurred while clamping the specimens to the tensile stress equipment or removing them from the hot plates. Burst test confirmed the existence of a plateau at about $8.3 \pm 1.6 \mathrm{MPa}$, a pressure much higher than those required for the great majority of microfluidics applications. The burst pressure 


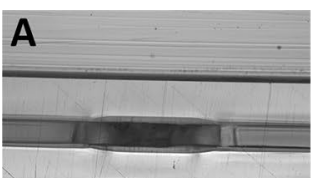

5 tons, $2 \mathrm{~min}, 70^{\circ} \mathrm{C}$

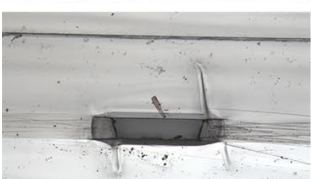

8 tons, $2 \mathrm{~min}, 70^{\circ} \mathrm{C}$
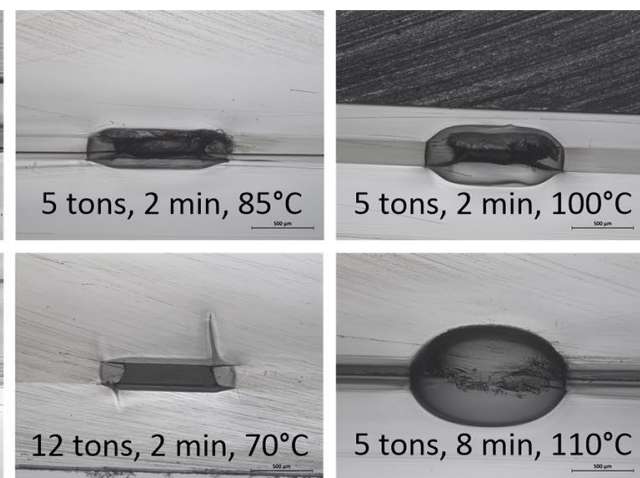
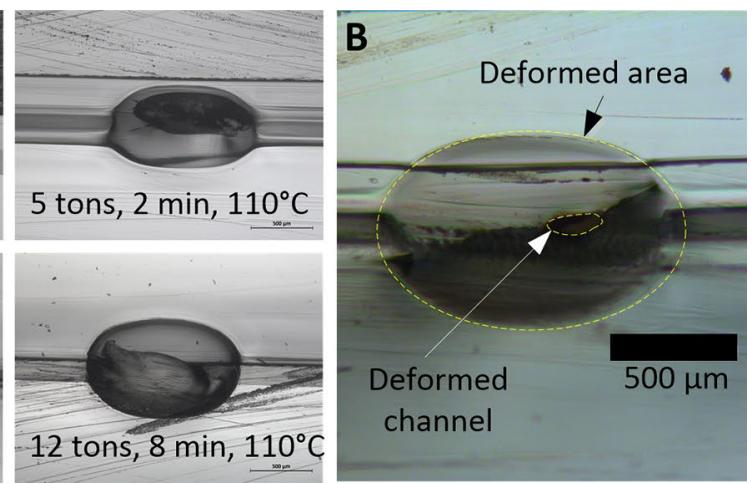

Fig. 3 a Microscope photographs of the channel cross sections showing the deformation in and around the channels at different bonding conditions. b Cross section of a channel bonded at $110{ }^{\circ} \mathrm{C}$,
2 min, 5 tons: as the real channel sags to a tenth of the initial area, the solvent changes the morphology of a large surrounding area

part of it before pressure was applied to the system. The evaporation of the solvent affects the burst test specimens to a lesser extent, as they are bonded on a much larger area, thus trapping ethanol within the layers to be bound and offering less air-ethanol contact surface available for evaporation to occur. Deformation analysis at $110{ }^{\circ} \mathrm{C}$ showed a $90 \%$ reduction in the cross-sectional area of the channels (Fig. 3b). In contrast, a deformed ellipsoidal zone is clearly visible, with area 2.5 times larger than initial channel. Complete channel disappearance and maximum deformation (nearly $400 \%$ in height) were obtained for samples bound at $110{ }^{\circ} \mathrm{C}$ for $8 \mathrm{~min}$, irrespective of whether the pressure was 5 or 12 tons at the ram (1.57 or $3.67 \mathrm{MPa}$ on specimen) (Fig. 3a).

\subsection{Comparison of different brands of PMMA}

PMMA sheets are mainly produced using two methods, casting or extrusion. While extrusion produces PMMA sheets with better thickness tolerances, cast PMMA has higher thermal stability and optical properties. Depending on the manufacturers, PMMA mechanical and chemical properties such as molecular weight and degree of polymerisation can vary within a certain range and may affect the bonding optimisation. Here we show how our optimal parameters $\left[70{ }^{\circ} \mathrm{C}, 5\right.$ tons $(1.57 \mathrm{MPa}$ on specimen), $2 \mathrm{~min}$ ] perform with PMMA supplied by different manufacturers, without further optimisation. Interestingly, Perspex ${ }^{\circledR}$ PMMA, no matter if cast or extruded, performed in the same way as Clarex ${ }^{\circledR}$ PMMA and shear stress results gave nearly overlapping bonding strengths. On the other hand, several cracks were noticed on the specimens cut-off extruded sheets. Also, this material showed the tendency to arch and deform during laser cutting, probably due to the relaxation of stress induced during manufacturing. Oroglass ${ }^{\circledR}$ PMMA showed lower bonding strength compared to the others. These results suggest that the applicability of our temperature must have lost, by evaporation, a considerable 


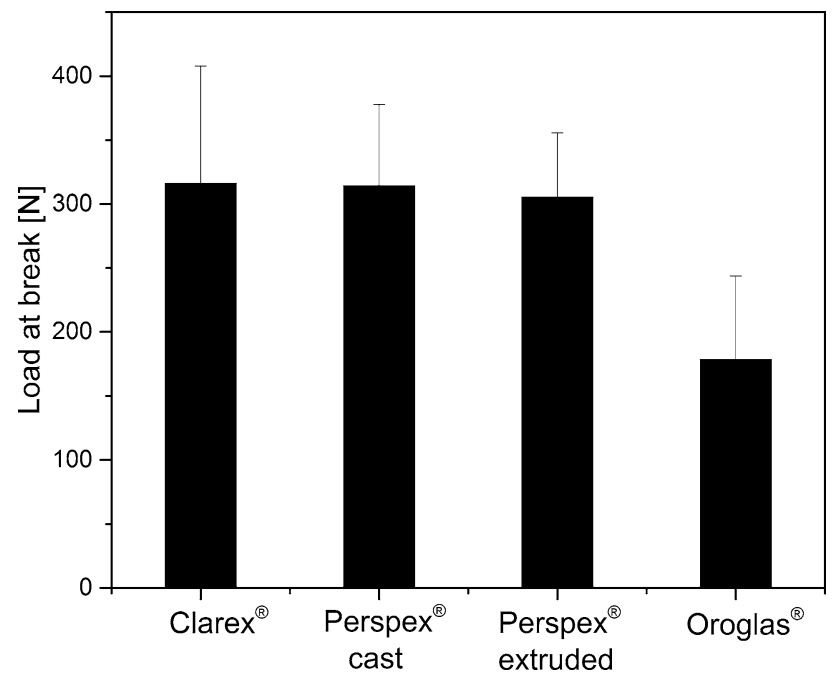

Fig. 4 Comparing PMMA from various manufacturers. To test the applicability of the technique to multiple brands of PMMA, Instron shear stress testing was performed on specimens machined from PMMA sheets $\left[N=9\right.$ for all samples, but Perspex ${ }^{\circledR}$ cast $(N=7)$ and Clarex $\left.^{\circledR}(N=12)\right]$. Error bars show the SD

method does not depend on the manufacturing process (cast or extruded) used, but rather on the grade of the polymer. Long polymer chains require greater energy to move than shorter ones; thus, the bonding of higher molecular weight $\left(M_{\mathrm{w}}\right)$ PMMA will require longer time and higher amount of solvent to be as efficient as the bonding of lower $M_{\mathrm{w}}$ PMMA. Since Oroglass ${ }^{\circledR}$ material reports higher mechanical and thermal properties (see Table.S1 Suppl. Inf.) than the others, it is legitimate to assume that it is composed of a heavier polymer (Nguyen and Kausch 1999) which would explain why it is harder to create a bound using the same bonding conditions. Unfortunately, the different suppliers have not yet replied to our questions regarding their polymer composition, and our hypothesis remains unconfirmed. Nonetheless, it must be noticed that complete bonding was achieved for the whole surfaces treated regardless of the brand, thus showing the general applicability of the method (Fig. 4).

\subsection{Bonding stability and quickened ageing}

Quickened ageing tests were performed to verify bonding stability under extreme conditions. Statistical analysis shows that there is no significant difference between untreated specimens and those aged at $90{ }^{\circ} \mathrm{C}$ in dry conditions or in water at $37.5^{\circ} \mathrm{C}$ for $216 \mathrm{~h}$, and differences in the mean can be related to statistical variability alone $(p=0.87$ and 0.19 , respectively). These results, shown in Fig. 5, beside holding good promises for long-lasting shelf life (even if a thorough characterisation for longer times would be necessary), show that microfluidic chips bonded using

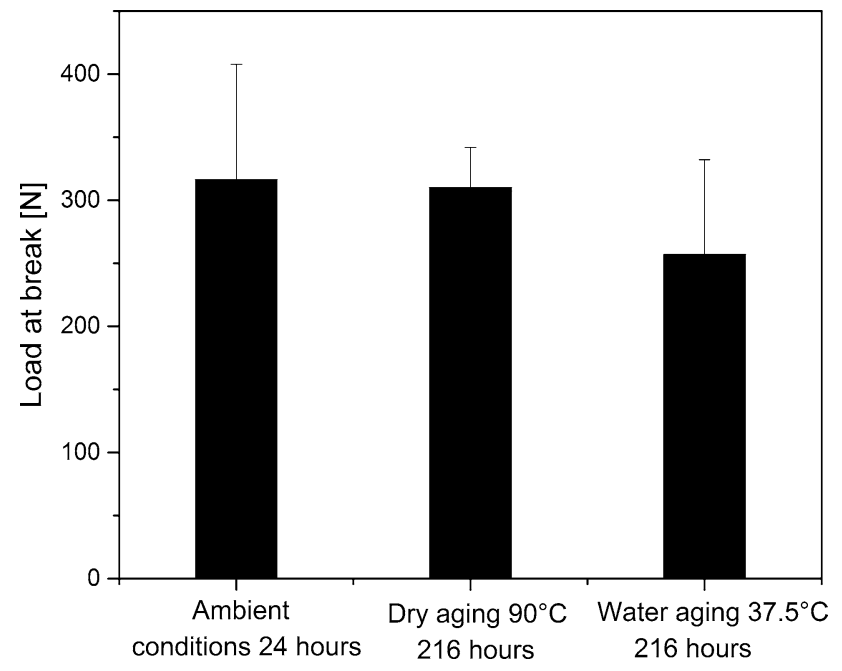

Fig. 5 Bonding stability at different environmental conditions. Instron shear stress testing was performed on specimens bonded at optimal conditions and then aged at $90{ }^{\circ} \mathrm{C}$ in dry conditions or $37.5^{\circ} \mathrm{C}$ in water, for $216 \mathrm{~h}$. Error bars show the standard deviation. There are no statistical differences between the treated and untreated specimens (respectively, $p=0.87$ and 0.19 ). $N=9$ for all samples but ambient conditions $N=12$

this method can be applied to several different biological studies, including cell-related investigations, where $37.5^{\circ} \mathrm{C}$ water incubation is frequent, and even on-chip PCR which involves temperature cycling up to about $95^{\circ} \mathrm{C}$.

\subsection{Microfluidic device examples}

To prove the reliability of the bonding protocol to common microfluidic tasks, several typical microfluidic applications are demonstrated. All the devices were produced by designing their layers on AutoCad (Autodesk, UK) and laser cutting them from sheets of PMMA with thicknesses $0.2,0.8$ or $2 \mathrm{~mm}$.

\subsubsection{Five-layer split-and-recombine mixer}

A five-layer split-and-recombine mixer was realised and tested under a range of flow rates. The bottom layer (\#1) was realised in 2.0-mm-thick PMMA, to allow for microfluidic connections. The other four layers (\#2, 3, 4 and 5) were realised in 0.2-mm-thick PMMA (Fig. 6a.i). The average channel cross section was $200 \mu \mathrm{m} \times 500 \mu \mathrm{m}$. The bonding step was realised with the optimal parameters $70{ }^{\circ} \mathrm{C}, 1.57 \mathrm{MPa}, 120 \mathrm{~s}$ (respectively bonding temperature, pressure and time). Two syringe pumps (World Precision Instruments, UK) were used to drive blue and yellow food dyes mixed with deionised water through the device. Flow rates (always equal for both syringe pumps) ranged from 10 up to $170 \mathrm{ml} / \mathrm{h}$ (upper limit for the pumps used) (Fig. 6a. ii). Although the chip shown here simply mixes two liquid streams, it proves that leak-proof 3D microfluidic structures 

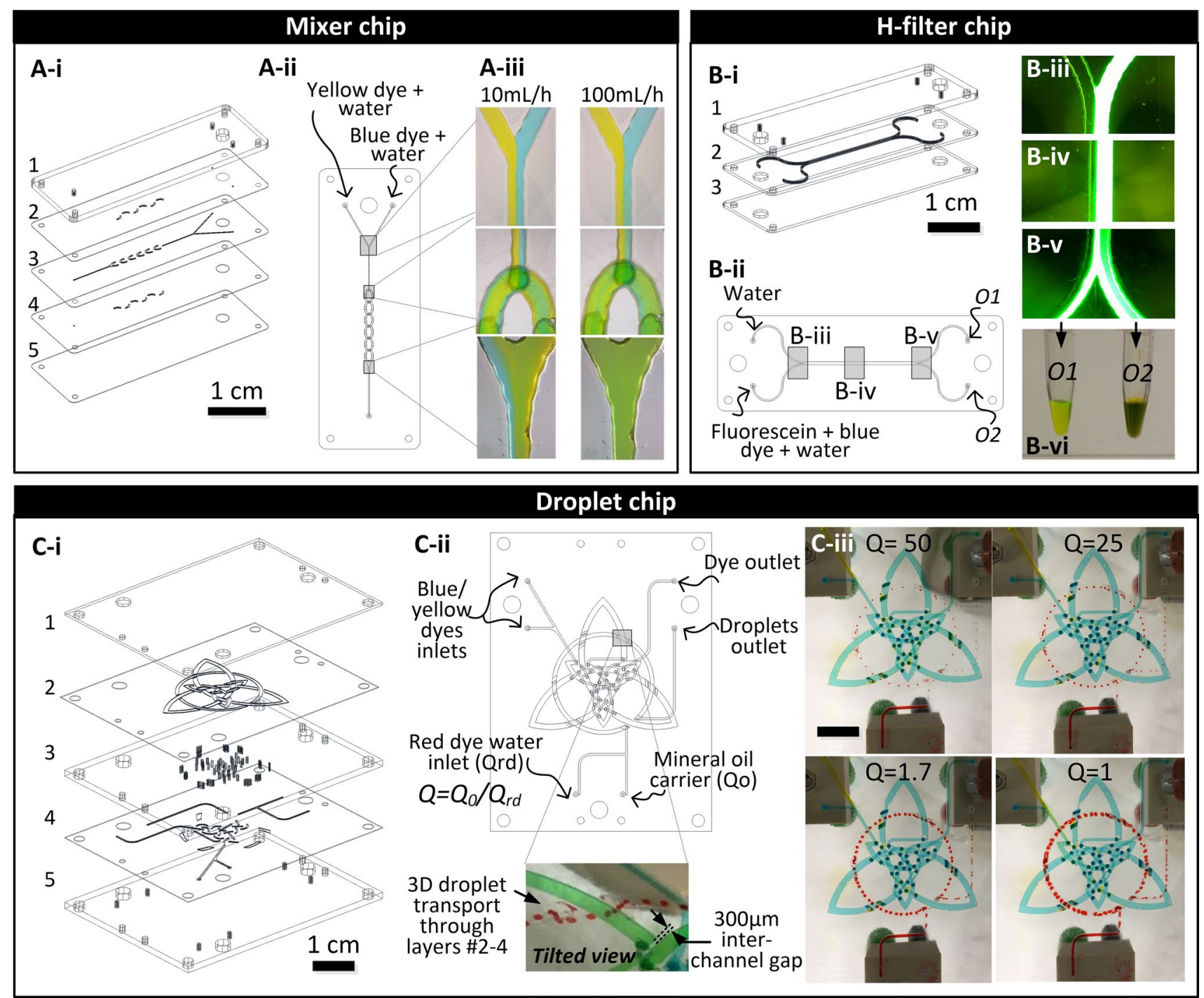

Fig. 6 Practical examples of microstructures manufactured using the proposed ultrafast method. a Five-layer split-and-recombine mixer test chip. a-i Blow-up view of the chip design. The chip has the footprint of a microscope slide $(75 \times 25 \mathrm{~mm})$ and is composed of four 0.2-mm PMMA layers and one 2.0-mm PMMA layer (bottom layer). a-ii Top view of the assembly. The grey zones correspond to the visualisation windows: a-iii Mixing patterns obtained at 10 and $100 \mathrm{ml} / \mathrm{h}$ at three locations on the chip. b $\mathrm{H}$ filter chip. b-i Blow-up view of the chip design. B-ii Top view of the $\mathrm{H}$ filter chip. The grey zones cor-

can be realised in a single bonding step. The five-layer mixer showed no sign of clogging nor leaking for any of the flow rate used $(10 \mathrm{ml} / \mathrm{h}$ up to the maximum pump rate of $170 \mathrm{ml} / \mathrm{h}$ ), and bonding of the layers is consistent along the entire surface. Mixing of the two streams was observed under microscope at three locations on the device (Fig. 6b). As expected, mixing was more efficient at higher flow rates (Fig. 6a.iii and iv, respectively, $10 \mathrm{ml}$ and $100 \mathrm{ml} / \mathrm{h}$ ). Supplementary Information video shows the complete manufacturing and testing of this device. respond to the visualisation windows: b-iii-b-vi shows the result of the separation in two Eppendorf tubes. $\mathbf{c}$ Droplet generation and celtic design pattern. c-i Blow-up view of the five-layer chip. c-ii Top view of the assembly with inlets and outlets. The inset shows a tilted view of the droplet channel passing underneath one of the branches of the triquetra as well as a 300- $\mu \mathrm{m}$ interchannel gap. c-iii Photographs of the device with four flow rate ratios producing four different droplet sizes

\subsubsection{Three-layer H filter}

A three-layer $\mathrm{H}$ filter (Fig. 6b-i) was realised and used to demonstrate the separation of fluorescein from blue food dye. The design features inlet and outlets channels placed $3 \mathrm{~mm}$ from the edges of the chip (Fig. 6b-ii), and with this feature we intended to demonstrate that the bonding obtained with this method is reliable along the entire surface of the chip, even close to the edges of the chip. The length of the main channel was $30 \mathrm{~mm}$, and the width on 
drawing was $1 \mathrm{~mm}$. A 3-ml syringe filled with deionised water was loaded on the first inlet, while a 3-ml syringe was filled with a mixture of fluorescein sodium salt (Sigma-Aldrich, $26 \mathrm{mg} / \mathrm{ml}$ ), blue food dye (Classikool, $5 \% \mathrm{v} / \mathrm{v})$ and deionised water. The syringe was fitted on syringe pumps and actuated at $1.5 \mathrm{ml} / \mathrm{h}$. Images were taken using a Dino-Lite (AM4113T-YFGW, Dino-Lite Europe, Naarden, Netherlands) under UV excitation (Fig. 6b-iii, iv and v). Fluorescein having a higher diffusion coefficient $\left(5.4 \times 10^{-4} \mathrm{~cm}^{2} / \mathrm{min}\right)$ than typical natural blue dyes $\left(4.942 \times 10^{-6} \mathrm{~cm}^{2} / \mathrm{min}\right.$ (Kawahito and Yasukawa 2009)) can be separated across the length of the channel to outlet $\# \mathrm{O} 1$ while the food dye does not diffuse into the water stream and exits exclusively in outlet \#O2. Figure 6c-vi shows the result of the extraction.

\subsubsection{Droplet chip}

Another five-layer device was created to demonstrate the intricacy of designs permitted by this technique as well as the reliability of the bonding for droplet applications (Fig. 6c-i). A microfluidic double triquetra (classic symbol in several European cultural groups) was created. Droplets are produced at the intersection of two channels merging in a circular channel surrounding the triquetra symbol and passing above and underneath the successive branches of the triquetra (inset, Fig. 6c-ii). Two inlet channels branching in the channel at a $90^{\circ}$ angle allow the creation of droplets by flowing mineral oil at flow rate $\mathrm{Q}_{0}$ and aqueous coloured solution at flow rate $Q_{\mathrm{rd}}$. We demonstrate the production of various droplet sizes using different flow rate ratio $Q=Q_{0} / Q_{\mathrm{rd}}$ (Fig. 6c iii, iv, v and vi, respectively, $\mathrm{Q}=50,25,1.7$ and 1$)$.

\subsubsection{Nineteen-layer assembly}

For proof of concept, we designed a nineteen-layer London tube map, featuring 9 of the tube lines adapted to fit the present layout (Jubilee, Bakerloo, Metropolitan, Hammersmith, Northern, Picadilly, Central, Circle and District). The chip measures $50 \times 50 \mathrm{~mm}$, with channel widths on drawing, $200 \mu \mathrm{m}$. Each layer is $200 \mu \mathrm{m}$ (total stack thickness is $3.8 \mathrm{~mm}$ ). Each London tube line is filled with a different coloured dye corresponding to its official colour as illustrated in Fig. 7. This proof-of-concept chip was cut and assembled in minutes and thus demonstrates the ease and flexibility of the technique with regard to the number of layers that can be assembled in one single step. Finally we also demonstrated the use of this technique in the context of public engagement, school kid activities and undergraduate teaching (Bridle et al. 2016; Morton and Bridle 2016).

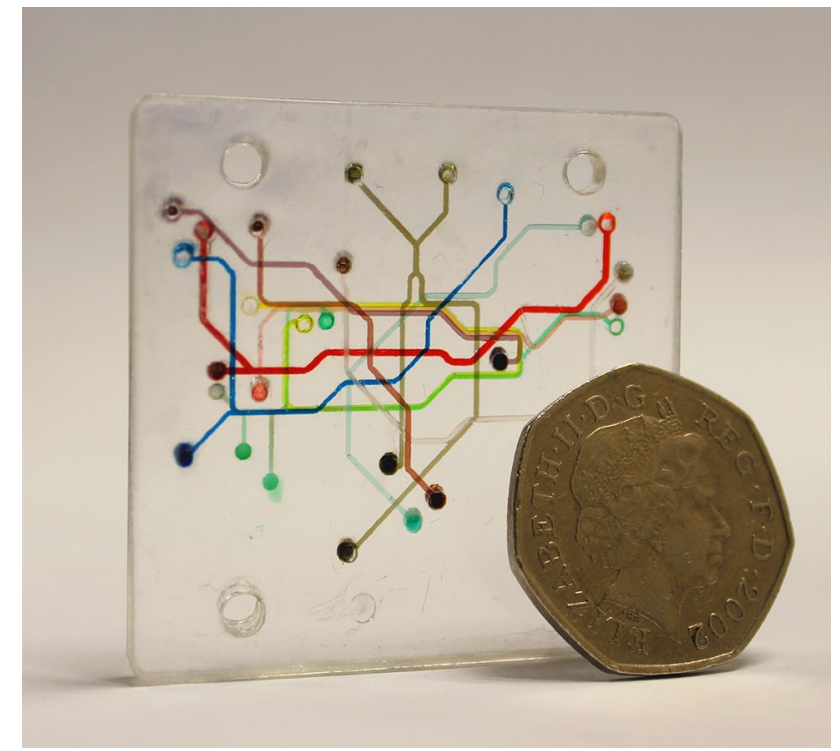

Fig. 7 Microfluidic London tube map. Proof-of-concept, nineteenlayer assembly representing nine of the London tube lines. The assembly of this chip was performed in a single step at the optimum time, pressure and temperature. $250 \mu \mathrm{l}$ of ethanol corresponding to our recommend volume of $10 \mu \mathrm{l} / \mathrm{cm}^{2}$ was applied directly on the side of the stack prior to mounting the stack on the press. Each tube line is filled with diluted food dye corresponding to its official colour [respectively, Jubilee (grey), Bakerloo (brown), Metropolitan (purple), Hammersmith (light pink), Northern (black), Victoria (light blue), Piccadilly (blue), Central (red), Circle (yellow) and District (green)] (colour figure online)

\subsection{Comparison with existing techniques}

In Table 1, our method is compared with the most recent advancements in PMMA bonding. The only method which can be performed more quickly (Toossi et al. 2015) requires the deposition of a gold layer to allow microwave heating at the surfaces. The present technique is the fastest and presents deformation in the lowest range and is the only technique that has been demonstrated with up to 19 PMMA layers. Additionally our method only requires a hot press and an inexpensive as well as innocuous solvent. Nonetheless, this method was always employed for channels with dimensions of $100 \mu \mathrm{m}$ or more and may not be as suitable for nanochannels as some of the other techniques listed. No channel clogging was ever encountered for channel cross sections higher or equal to $200 \mu \mathrm{m} \times 200 \mu \mathrm{m}$; nonetheless, clogging might occur for smaller channel width due to the small solvent erosion effect discussed previously. Therefore, the bonding technique is not suggested when features smaller than $100 \mu \mathrm{m}$ are required. In comparison with 3D printing, another rapid prototyping method which potentially alleviates the need for bonding and produces similar results for channel above $100 \mu \mathrm{m}$ (Comina et al. 2015; 
Table 1 Comparisons of thermal techniques for bonding PMMA

\begin{tabular}{|c|c|c|c|c|c|c|}
\hline Method & Bonding time & $T^{\circ}\left({ }^{\circ} \mathrm{C}\right)$ & $\begin{array}{l}\text { Bonding strength } \\
\text { (method) }\end{array}$ & Deformation & $\begin{array}{l}\text { Maximum number of } \\
\text { layers demonstrated }\end{array}$ & Refs. \\
\hline $\begin{array}{l}\text { Oxygen plasma treat- } \\
\text { ment }\end{array}$ & $>25 \min$ & 85 & $0.57 \mathrm{MPa}$ (tensile test) & $\begin{array}{l}<1 \% \text { for microchan- } \\
\text { nels, } 10 \% \text { on nano- } \\
\text { channels }\end{array}$ & 2 & Zhifu et al. (2015) \\
\hline Plasticiser-assisted $^{\mathrm{a}}$ & $>20 \min$ & 90 & N.C. & $<10 \%$ & 4 & Duan et al. (2010) \\
\hline Acetonitrile-assisted & $<15 \min$ & 25 & N.C. & N.C. & 2 & Sun et al. (2007) \\
\hline Ultrasound treatment & $5 \min$ & 70 & $\begin{array}{l}0.7 \mathrm{~J} / \mathrm{cm}^{2} \text { (crack open- } \\
\mathrm{ing} \text { ) }\end{array}$ & $5 \%$ on nanochannels & 2 & Zhang et al. (2010) \\
\hline In-mold $^{b}$ & $4 \min$ & 100 & $0.35 \mathrm{MPa}$ & $<10 \%$ & 2 & Chu et al. (2015) \\
\hline Microwave $^{c}$ & $35 \mathrm{~s}$ & $50-160$ & 1.4 MPa (tensile test) & $<5 \%$ & 2 & Toossi et al. (2015) \\
\hline $\begin{array}{l}\text { Ultrafast } \mathrm{C}_{2} \mathrm{H}_{6} \mathrm{O}- \\
\text { assisted }\end{array}$ & $2 \min$ & 70 & 1.23 MPa (shear test) & $<5 \%$ & 19 & \\
\hline
\end{tabular}

Morgan et al. 2016), our combined laser cutting and ethanol-assisted bonding method is faster and more flexible. To produce the mixer example chip (Fig. 6a) on a typical high-resolution 3D printer such as the multimaterial polyjet CONNEX 500 would take approximately $45 \mathrm{~min}$, printing only. The resulting product would then need to be soaked overnight to remove the support material in the intricate feature of the chip. Alternatively, the chip would be printed in several parts and then bonded, creating the same situation as the current method.

\section{Conclusions}

Ethanol-assisted thermal bonding of PMMA was optimised and characterised for the purpose of microfluidic prototyping. This method was demonstrated in conjunction with laser cutting, but is adaptable to other structuring methods such as moulding, hot embossing, stamping and milling, and is therefore also relevant to mass production. We showed that it is possible to reach a shear bonding strength of at least $1.5 \mathrm{MPa}$ (dividing the load at break for the whole bonded area), and channels able to withstand pressures over $6.2 \mathrm{MPa}$ (minimum burst pressure recorded), by pressing PMMA layers for just 2 min at $70{ }^{\circ} \mathrm{C}$ and $1.57 \mathrm{MPa}$. These conditions are very mild on the material, the temperature being $40{ }^{\circ} \mathrm{C}$ lower than PMMA glass transition, and easy to reach, since the pressure required is in the lowest range of any hydraulic press. Bonding stability was demonstrated with quickened ageing at $90{ }^{\circ} \mathrm{C}$ (dry conditions) and $37.5{ }^{\circ} \mathrm{C}$ in water for $216 \mathrm{~h}$. Several layers can be stacked together in one bonding step (up to 19 have been demonstrated), creating three-dimensional structures. The channel height deformation observed is always lower than $5 \%$. We demonstrated the manufacture of several structures including mixers, $\mathrm{H}$ filter, droplet generators capable of sustaining flow rates of at least $170 \mathrm{ml} / \mathrm{min}$, which is sufficient for most microfluidic applications, including high flow rate applications. This ultrafast technique is applicable to microfluidic devices with minimum feature size of $150 \mu \mathrm{m}$ and requires a low capital investment of around $\$ 16,000$ (including laser cutter and bonding equipment). It will be particularly attractive within microfluidic laboratories which are looking for rapid translation of devices into products, as well as for rapid prototyping, and the educational aspects of this technique will be of interest to a broader public, such as secondary, undergraduate and postgraduate teaching professionals and more generally to developers and researchers requiring multilayer, polymeric and cost-effective solutions.

Acknowledgements AL is funded by a Heriot-Watt James Watt PhD studentship. MKK acknowledges the Royal Academy of Engineering for funding. We thank Prof. Marc Desmulliez for early access to laser cutting equipment. We thank Dr. Helen Bridle and Ewa Guźniczak for access to the Nemesys equipment. We thank Prof. Jason Hong and Dr Jia Ni for related to the thermal cycling equipment. An H. Tran took the photographs of the droplet generation chip and the nineteen-layer assembly London tube map. We would like to thank the anonymous reviewers for their constructive comments.

Open Access This article is distributed under the terms of the Creative Commons Attribution 4.0 International License (http://creativecommons.org/licenses/by/4.0/), which permits unrestricted use, distribution, and reproduction in any medium, provided you give appropriate credit to the original author(s) and the source, provide a link to the Creative Commons license, and indicate if changes were made. 


\section{References}

Baldan A (2012) Adhesion phenomena in bonded joints. Int J Adhes Adhes 38:95-116

Becker H (2010) Mind the gap! Lab Chip 10(3):271-273

Bird RB, Stewart WE, Lightfoot EN (2002) Transport phenomena, 2nd edn. Wiley, New York

Bridle H, Morton J, Cameron P, Desmulliez MPY, Kersaudy-Kerhoas M (2016) Design of problem-based learning activities in the field of microfluidics for 12- to 13-year-old participants-Small Plumbing!: empowering the next generation of microfluidic engineers. Microfluid Nanofluid 20(7):1-11

Brown L, Koerner T, Horton JH, Oleschuk RD (2006) Fabrication and characterization of poly(methylmethacrylate) microfluidic devices bonded using surface modifications and solvents. Lab Chip 6(1):66-73

Cheng E, Yin Z, Zou H, Chen L (2015) Surface modification-assisted bonding of 2D polymer-based nanofluidic devices. Microfluid Nanofluid 18(3):527-535

Chu C, Jiang B, Zhu L, Jiang F (2015) A process analysis for microchannel deformation and bonding strength by in-mold bonding of microfluidic chips. J Polym Eng 35:267

Comina G, Suska A, Filippini D (2015) 3D printed unibody lab-ona-chip: features survey and check-valves integration. Micromachines 6(4):437

Das D, Duraiswamy S, Yi Z, Chan V, Yang C (2015) Continuous droplet-based liquid-liquid extraction of phenol from oil. Sep Sci Technol 50(7):1023-1029

Duan H, Zhang L, Chen G (2010) Plasticizer-assisted bonding of poly(methyl methacrylate) microfluidic chips at low temperature. J Chromatogr A 1217(1):160-166

Frot C, Taccoen N, Baroud CN (2016) Frugal droplet microfluidics using consumer opto-electronics. PLoS ONE 11(8):e0161490

Green DW, Perry RH (2008) Perry's chemical engineers' handbook, 8th edn. McGraw-Hill, New York

Hardt S, Drese KS, Hessel V, Schönfeld F (2005) Passive micromixers for applications in the microreactor and $\mu$ TAS fields. Microfluid Nanofluid 1(2): 108-118

Holmes RJ, McDonagh C, McLaughlin JAD, Mohr S, Goddard NJ, Fielden PR (2011) Microwave bonding of poly(methylmethacrylate) microfluidic devices using a conductive polymer. J Phys Chem Solids 72(6):626-629

Hsu Y-C, Chen T-Y (2007) Applying Taguchi methods for solventassisted PMMA bonding technique for static and dynamic $\mu$-TAS devices. Biomed Microdevices 9(4):513-522

Jiang J, Zhan J, Yue W, Yang M, Yi C, Li C-W (2015) A single lowcost microfabrication approach for polymethylmethacrylate, polystyrene, polycarbonate and polysulfone based microdevices. RSC Adv 5(45):36036-36043

Kalkandjiev K, Gutzweiler L, Welsche M, Zengerle R, Koltay P (2010) A novel approach for the fabrication of all-polymer microfluidic devices. In: Proceedings of the IEEE international conference on micro electro mechanical systems (MEMS)

Kawahito M, Yasukawa R (2009) Characteristics of color produced by Awa natural indigo and synthetic indigo. Materials 2(2):661-673

Klank H, Kutter JP, Geschke O (2002) $\mathrm{CO}_{2}$-laser micromachining and back-end processing for rapid production of PMMA-based microfluidic systems. Lab Chip 2(4):242-246

Li L, Chen J, Deng W, Zhang C, Sha Y, Cheng Z, Xue G, Zhou D (2015) Glass transitions of poly(methyl methacrylate) confined in nanopores: conversion of three- and two-layer models. J Phys Chem B 119(15):5047-5054

Lin C-H, Chao C-H, Lan C-W (2007) Low azeotropic solvent for bonding of PMMA microfluidic devices. Sens Actuators B Chem 121(2):698-705
Madadi H, Mohammadi M, Casals-Terre J, Lopez RC (2013) A novel fabrication technique to minimize poly(dimethylsiloxane)microchannels deformation under high-pressure operation. Electrophoresis 34(22-23):3126-3132

Mohammed MI, Abraham E, Desmulliez MPY (2013) Rapid laser prototyping of valves for microfluidic autonomous systems. J Micromech Microeng 23(3):035034

Mona R, Sumanpreet C, Dan S, Parameswaran M (2010) Microwaveinduced, thermally assisted solvent bonding for low-cost PMMA microfluidic devices. J Micromech Microeng 20(1):015026

Morgan AJ, San Jose LH, Jamieson WD, Wymant JM, Song B, Stephens P, Barrow DA, Castell OK (2016) Simple and versatile 3D printed microfluidics using fused filament fabrication. PLoS ONE 11(4):e0152023

Morton JAS, Bridle H (2016) Student-led microfluidics lab practicals: improving engagement and learning outcomes. Biomicrofluidics 10(3):034117

Nascimento S, Pólvora A (2016) Maker cultures and the prospects for technological action. Sci Eng Ethics. doi:10.1007/s11948-016-9796-8

Nayak NC, Yue CY, Lam YC, Tan YL (2009) Thermal bonding of PMMA: effect of polymer molecular weight. Microsyst Technol 16(3):487-491

Nguyen T, Kausch H (1999) Molecular weight distribution and mechanical properties: mechanical properties and testing of polymers. Mol Weight Distrib Mech Prop 3:7

Pagaduan JV, Ramsden M, O'Neill K, Woolley AT (2015) Microchip immunoaffinity electrophoresis of antibody-thymidine kinase 1 complex. Electrophoresis 36(5):813-817

Romoli L, Tantussi G, Dini G (2011) Experimental approach to the laser machining of PMMA substrates for the fabrication of microfluidic devices. Opt Lasers Eng 49(3):419-427

Santos CIAV, Mialdun A, Legros JC, Shevtsova V (2016) Sorption equilibria and diffusion of toluene, methanol, and cyclohexane in/through elastomers. J Appl Polym Sci 133(21):43449

Silva R, Heuckroth A, Huang C, Rolfe A, Densmore D (2015) MakerFluidics: microfluidics for all. Synberc: Fall 2015

Sun X, Peeni BA, Yang W, Becerril HA, Woolley AT (2007) Rapid prototyping of poly(methyl methacrylate) microfluidic systems using solvent imprinting and bonding. J Chromatogr A 1162(2):162-166

Toossi A, Moghadas H, Daneshmand M, Sameoto D (2015) Bonding PMMA microfluidics using commercial microwave ovens. J Micromech Microeng 25(8):85008-85018

Tran HH, Wu W, Lee NY (2013) Ethanol and UV-assisted instantaneous bonding of PMMA assemblies and tuning in bonding reversibility. Sens Actuators B Chem 181:955-962

Tsao C-W, DeVoe DL (2009) Bonding of thermoplastic polymer microfluidics. Microfluid Nanofluid 6(1):1-16

Wan AMD, Sadri A, Young EWK (2015) Liquid phase solvent bonding of plastic microfluidic devices assisted by retention grooves. Lab Chip 15(18):3785-3792

Wang P-P, Lee S, Harmon JP (1994) Ethanol-induced crack healing in poly(methyl methacrylate). J Polym Sci, Part B: Polym Phys 32(7):1217-1227

Yang K (2014) Gravitational field-flow fractionation devices fabricated via a hot embossing/thermal bonding method. Micromachines 5(2):139-153

Zhang Z, Luo Y, Wang X, He S, Meng F, Wang L (2010) Bonding of planar poly (methyl methacrylate) (PMMA) nanofluidic channels using thermal assisted ultrasonic bonding method. Microsyst Technol 16(12):2043-2048

Zhifu Y, Lei S, Helin Z, Cheng E (2015) Two dimensional PMMA nanofluidic device fabricated by hot embossing and oxygen plasma assisted thermal bonding methods. Nanotechnology 26(21):215302

Zhu X, Liu G, Guo Y, Tian Y (2006) Study of PMMA thermal bonding. Microsyst Technol 13(3):403-407 